\title{
REQUISITOS DEL "NON USUS" EN LA EXTINCION DE LAS SERVIDUMBRES
}

FERMIN CAMACHO DE LOS RIOS

Prof. Titular de Derecho Romano

El Derecho Romano, tan acorde en sus principios, hace que las servidumbres se extingan mediante actos jurídicos contrarios a aquellos que generaron su constitución (1), su "bestellung" (2).

Como sabemos, en el ius civile con frecuencia el tanscurso del tiempo puede hacer que alguien adquiera un derecho, pero no que lo pierda. En general el paso del tiempo, la prescripción, no es causa habitual de extinción. Inicialmente las acciones procedentes del ámbito civil no están sujetas a prescripción (3), ni los derechos -aún más los reales- suelen extinguirse por el mero hecho de no ejercitarlos.

En realidad los derechos reales sobre cosa ajena no se pueden perder porque no sean ejercitados, sino indirectamente como consecuencia de que otro, mediante prescripción, adquiera la propiedad de la misma cosa sin gravámenes. Sólo en este sentido la prescripción implica extinción, desde el día en que se ha cumplido, tanto del derecho como de la acción que lo defiende.

En verdad, el no uso (4) de los derechos reales y la prescripción son instituciones bien diferentes (5). El non usus, por vía de la más antigua interpretación de la usucapión, fue admitido como un modo útil para extinguir las servidumbres (6). Cuando la servidumbre no se ejercitaba durante el tiempo indicado para hacer válida la usucapión, el propietario del fundo, una vez que lo había poseido libre de cargas durante dicho plazo temporal, adquiere a través de la usucapión el mismo fundo, pero ahora como libre, extinguiendo la servidumbre.

De aquí que se hable en las servidumbres urbanas de usucapio libertatis, para indicar que el no uso genera el hecho adquisitivo de la libertad de la carga (7).

La servidumbre sólo se pierde como consecuencia de una adquisición; cuando mediante usus se haya adquirido de facto una situación incompatible con un derecho ya existente, que sucumbe frente a la adquisición.

La causa de la extinción no es intrínsecamente el non usus, sino el usus paralelo de la cosa. De nuevo los derechos no se pierden por su falta de uso, sino porque otros los hayan adquirido poseyéndolos.

La prescripción, fruto evidente del derecho pretorio, hace que determinadas acciones puedan ejercitarse sólo en un breve período de tiempo, en general un año para las honorarias, como sabemos, un plazo no dispuesto para el no uso (8).

Fue Teodosio II, en el 424, quien indicó de forma estable el plazo de treinta años para la prescripción de todas las acciones. CT 4, 14, 1 (9):

Sicut in re speciali est, ita ad universitatem ac personales actiones ultra triginta annorum spatium minime protendantur. Sed si qua res 
vel ius aliquod postuletur vel persona qualicumque actione vel persecutione pulsetur, nihilo minus erit agenti triginta annorum praescriptio metuenda: eodem etiam in eius valente persona, qui pignus vel hypothecam non a suo debitore, sed ad alio possidente nititur vindicare.

Justiniano sin embargo, superando la disposición de las XII Tablas que nos indicaba usus auctoritas fundi biennium ceteram rerum annus esto, en C.I. 3, 34,13 (10) sancionó que las servidumbres se extinguen por non usus en el plazo de diez años entre presentes o veinte entre ausentes. Distinción no contemplada en nuestro actual Código Civil (11). Evidentemente la diferencia en la determinación del plazo temporal no es gratuita, y ns pone una vez más de manifiesto la separación entre non usus y prescriptio" (12).

Lo cierto es que en ningún párrafo de la Compilación justinianea se hace la más mínima similitud entre el no uso y la prescripción, ni siquiera de forma implícita. El no uso se configura como un régimen autónomo, sobre el que no influye la teoría general de la prescripción. Se trata de un modo particular de extinción, aún cuando sea asimilable, por los efectos que consuma, a la prescripción extintiva, de forma que las disposiciones relativas a ésta sólo serán aplicables al régimen jurídico del non usus en cuanto sean conformes a la naturaleza propia de los derechos reales a los que afecte.

Con todo, la realidad es que el derecho se perdía por el hecho de que no se usara por un bienio en el Derecho clásico, y por diez años entre presentes o veinte entre ausentes en el Derecho justinianeo, y así nos lo atestiguan las fuentes: Paulo en sus Sentencias III, 6,30 .

Justiniano modificó tal norma estableciendo que el usufructo, sea sobre cosas muebles como inmuebles, se extinguía por non usus en diez años, en dos de sus Qinquaginta Decisiones (C. 3, 33, 16 y C. 3, 34, 13). En otras palabras, Justiniano aplicó a la servidumbre la ordinaria usucapión, que era el fruto de la fusión de la antigua usucapio y de la longi temporis praescriptio (13).

Para que el no uso tenga esa capacidad extintiva es necesario un determinado no uso, cualificado con la concurrencia de determinados requisitos:

I. Debe comprobarse un efectivo no uso, es decir, una falta de ejercicio, por parte del titular del derecho o a quien este haya enajenado o cedido su ejercicio: el adquirente del uso, el donatario, el gestor de negocios, como nos indica D. $7,1,12$, 2 y D. $7,1,12,3$.

2. Usufructuarius vil ipse frui ea re, velalii fruendam concedere, vel locare, vel vendere postest; nam et qui locat, utitur, et qui vendit, donet, puto eum uti, atque ideo retineri usumfructum; et hoc Cassius et Pegasus responderunt, et Pomponius libro quinto ex Sabino porbat. Non solum autem si ego locavero, retineo usumfructum, sed en si alius negotium meum gerens locaverit usumfructum, Iulianus libro trigesimo quinto scripsit, retinere me usumfructum. Quid tamen, si non locavero, sed absente et ignorante me negotium meum gerens utatur quis et ignorante me negotium meum gerens utatur quis et fruatur? Nihilo minus retinero usumfructum. Quod et Pomponius libro quinto probat per hoc, quod negotiorum gestorum actionen acquisivi. 
3. De illo Pomponius dubitat, si fugitivus, in quo meus ususfructus est, stipuletur aliquid ex re mea, vel per traditionem accipiat, an per hoc ipsum, quasi utar, retineam usumfructum? Magisque admittit, retinere; nam saepe, etiamsi praesentibus servis non utamur, tamen usumfructum retinemus, utputa aegrotante servo, vel infante, cuius operae nullae sunt, vel defectae senectutis homine; nam et si agrum aremus, licet tam sterilis sit, ut nullus fructus nascatur, retinemus usumfructum. Iulianus tamen libro trigesimo quinto Digestorum scribit, etiamsi non stipuletur quid servus fugitivus, retineri tamen usumfructum; nam qua retione, inquit, retinetur a proprietario possessio, etiamsi in fuga servus sit, pari ratione etiam ususfructus retinetur.

Las personas a quienes fue cedido el ejercicio no importa que no usen el derecho si la cesión fue onerosa, algo imposible sin embargo cuando fue realizada a título gratuito, como en la donación o el precario.

Si la cesión del derecho fue onerosa, el titular original goza del precio que para él representa el uso; si por el contrario se realizó una cesión a título gratuito, como sería una donación, se necesita el ejercicio del donatario, ya que sin él faltará todo medio para sostener la existencia del uso. Gayo y Muciano son claros en estos matices (D. 7, 1, 38; D. 7, 1, 39; D. 7, 1, 40).

38. Non utitur usufructuarius, si nec ipse utatur, nec nomine eius alius, puta qui emit, vel qui conduxit, vel cui donatus est, vel qui negotium eius gerit. Plane illud interest, quod, si vendidero usumfructum, etiamsi emtor non utatur, videor usumfructum retinere.

39. Quia, qui pretio fruitur, non minus habere intelligitur, quam qui principali re utitur fruitur.

40. Quod si donavero, non alias retineo, nisi ille utatur.

Como confirma también el hecho que si el ejercicio del derecho fue alquilado y el propietario gravado vendía el fundo, el titular de la servidumbre la pierde cuando el adquirente la ejercita a nomine propio, a preguntas de Pomponio responde Ulpiano en D. 7, 4, 29 y 1 :

29. Pomponius quaerit, si fundum a me proprietarius conduxerit, aumque fundum vendiderit Seio non deducto usufructu, an usumfructum per emtorem retineam? Et ait, licet proprieterius mihi pensionem solverit, tamen usumfructum amitti, quia non meo nomine, sed suo fruitus est emtor. Teneri plane mihi ex locato proprietarium, quanti mea interfuit, di factum non esse; quamquam si a me conductum usumfructum quis alii locaverit, retinetur ususfructus; sed si proprietarius eum locasset suo nomine, dicendum amitti, non enim meo nomine fruitur colonus.

1. Non solum usumfructum amitti capitis minutione constat, sed et actionem de usufructu; et parvi refert, utrum iure sis constitutus usufructus, an vero tuitionePraetoris. Proinde traditus quoque usufructus, item in fundo vectigali vel superficie non iure constitutus capitis minutione amittitur. 
A mayor abundamiento, y a tenor de D. 8,6,19, 1, la ignorancia del titular no paralizaba la extinción por non usus, D. $41,3,4,27$ :

27. Si viam habeam per tuum fundum, et tu me ab ea vi expuleris, por longun tempus non utendo amittam vim, quia nec possideri intelligitur ius incorporale, nec de via quis, id est mero iure, detruditur,

A sensu contrario, para la conservación de la servidumbre no resulta necesario que ésta sea ejercitada por el titular, simplemente basta que alguien a él ligado la ejecute, ya sea el poseedor o el simple o el simple arrendatario del ejercicio, como nos recuerdan D. $8,6,5 ;$ D. $8,6,6$; D. $8,6,20$.

5. Servitus et per socium, et fructuarium, et bonae fidei possessorem nobis retinetur;

6. Nam satis est fundi nomine itum esse.

1. Si ego via, quae nobis per vicini fundum debebatur, usus fuero, tu autem constituto tempore cessaveris, an ius tuum amiseris? et e contrario, si vicinus, cui via per nostrum fundum debebatur, per meam partem ierit, egerit, tuam partem ingressus non fuerit, au partem tuam liberaverit? Celsus respondit, si divisus est fundus inter socios regionibus, quod ad survitutem attinet, quae ei fundo debebatur, perinde est, atque si ab initio duobus fundis debita sit; et sibi quisque dominorum usurpat servitutem, sibi non utendo deperdit, nec amplius in ea re causae eorum fundorum miscentur, nec fit ulla iniuria ei, cuius fundus servit, imo si quo melior, quoniam alter dominorum utendo sibi, non toti fundo, proficit.

20. Usu retinetur servitus, quum ipse, cui debetur, utitur, quive in possessione eius est, aut mercenarius, aut hospes, aut medicus, quive ad visitandum dominium venit, vel colonus, aut fructuarius.

II. El no uso debe ser completo. Si el titular de la servidumbre utiliza sólo en parte el derecho, la pérdida que se origina no es de carácter global (14) (D. 7, 1, 5 y D. 7, 4, 25 y 27), a no ser que se afirmara, en el acto de constitución de la srvidumbre, la intención del titular de gozar del objeto por entero y de forma total. D. 7, 4, 20:

20. Is, qui usumfructum habet, si tantum utatur, quia existimet se usum tantum habere, an usumfructum retineat? Et siquidem sciens se usumfructum habere, tantun uti velit, nihilo minus et frui videtur, si vero ignoret, puto eum amittere fructum; non enim ex eo, quod habet, utitur, sed ex eo, quod putavit se habere.

Son numerosos los ejemplos que nos ofrecen las fuentes para denotar el carácter extintivo del non usus parcial; en D. $8,3,18$ pr., cuando una servidumbre afecta a más de un fundo, si no se ejercita en uno de ellos, durante un plazo similar al dispuesto para la prescripción, la servidumbre se mantiene activa y latente en todos los demás fundos; al igual que si sólo se ejercitan algunas de las facultades atribuidas a la servidumbre es suficiente para no invalidar las restantes (D. 8,6,2).

En esta línea también se orienta la redacción de nuestro Código Civil en su art. 548 , el supuesto de una titularidad comunitaria en una servidumbre, "si el predio 
dominante perteneciere a varios en común, el uso de la servidumbre hecho por uno impide la prescripción respecto de los demás".

Caso distinto es que la causa del non usus sea porque la servidumbre es ejercitada en modo distinto al establecido en su constitución; esta es causa suficiente para su extinción. Son clásicos los ejemplos que nos ofrecen el D. 8, 6, 10 y D. 8, 6, 18 (15).

10. Si commune, fundum ego et pupillus haberemus, licet uterque non uteretur, tamen propter pupillum et ego viam retineo.

$S i$ is, qui nocturnam aquam habet, interdiu per constitutum ad amissionem tempus usus fuerit, amisit nocturnam servitutem, qua usus non est. Idem est in eo, qui certis horis aquaeductum habens aliis usus fuerit, nec ulla parte eaurm horarum.

18. Si quis alia aqua usus fuerit, quam de qua in servitute imponenda actum est, servitus amittitur.

Mucius Scaevola no deja dudas, para el jurista es clara la distinción entre la prescripción de la servidumbre (titularidad del derecho) y de la forma (modo diverso de ejercitarla), en el primer supuesto existe un no uso del derecho y en el segundo una irregularidad en la forma de uso.

En nuestro derecho actual "la forma respecto de la servidumbre vale tanto como manera de ejercitarla". Para el Código es clara la distinción entre la prescripción de la servidumbre (titularidad del derecho) y de la forma (modo de ejercitarla), en el primer supuesto existe un no uso del derecho y en segundo una irregularidad cualitativa en la forma de uso.

III. El no uso debe ser imputable y voluntario. Debe ser una renuncia autónoma. Será Ulpiano en D. 27, 9, 3, 5 y D. 7, 1, 12, 3 quien nos precise este matiz.

3. De illo Pomponius dubitat, si fugitivus, in quo meus ususfructus est, stipuletur aliquid es re mea, vel per traditionem accipiat, an per hoc ipsum, quasi utar, retineam usumfructum? Magisque admittit, retinere; nam saepe, etiamsi praesentibus servis non utamur, tamen usumfructum retinemus, utputa aegrotante servo, vel infante, cuius operae nullae sunt, vel defectae senectutis homine; nam et si agrum aremus, licet tam sterilis sit, ut nullus fructus nascatur, retinemus usumfructum. Iulianus tamen libro trigesimo quinto Digestorum scribit, etiamsi tamen usumfructum; nam qua ratione, inquit, retinetur a proprietario possessio, etiamsi in fuga servus sit, pari ratione etiam ususfructus retinetur.

5. Nec ususfructus alienari postest, etsi solus fuit ususfructus pupiIli. An ergo hic nec non utendo amittatur, si tutor causam praebuerit huius rei? Et manifestum est, restaurari debere. Sed si proprietatem habeat pupillus, non potest usumfructum vel usum alienare, quamvis Oratio nihil de usufructu loquatur. Simili modo dici potest, nec servitutem imponi posse fundo pupilli vel adolescentis, nec servitutem remitti; quod et in fundo dotali placuit.

En el primer texto se indica que no se pierde la servidumbre si no se puede usar 
de facto del siervo que era objeto del usufructo, a causa de su huida, enfermedad o simplemente por viejo o infante. Mientras que el segundo, en atención a la oración de Settimio Severo del 195 que limitaba la facultad de vender los bienes del pupilo por el tutor, se indica que de forma análoga el pupilo no pierde la titularidad de la servidumbre que su tutor no ejercita en su nombre (16).

Dos ejemplos contundentes de la ineludeble voluntariedad e imputabilidad del no uso en la extinción del derecho.

IV. Debe transcurrir por entero dentro del plazo de tiempo establecido por la Ley. Dos años en el Derecho Clásico a tenor de lo indicado en la Ley de las XII Tablas, y diez o veinte años en Derecho Justinianeo según C. I. 3, 34, 13.

Dos son los problemas que nos plantea este plazo temporal de extinción, el primero se presenta en cuanto a la interrupción del plazo establecido (17). En realidad sólo cabe la interrupción que nace de las peculiaridades intrínsecas del ejercicio de la servidumbre, la denominada interrupción natural (18). Paulo en D. 7, 4, 28 nos autoriza esta excepción:

28. Si usufructus alternis annis legetur, non posse non utendo eum amitti, quia plura sunt legata.

El jurista nos recuerda el supuesto del "usufructus alterius annis constituto" que en razón a la especial peculiaridad de su ejercicio, el derecho no se extingue por non usus, ya que cada año se constituye un usufructo especial.

El otro problema es conocer con exactitud el inicio del plazo extintivo. Para conocer desde qué día se comienza a computar el tiempo del non usus, debemos acudir al fundamento mismo del hecho extintivo, esto es, la paralela posesión que el propietario gravado realiza sobre el objeto de la servidumbre como libre de cargas. Desde que se ha comenzado a poseer el fundo como libre.

Evidentemente se debe considerar el carácter contínuo o discontínuo de la propia servidumbre, en las servidumbres denominadas contínuas el período se inicia desde el último acto de ejercicio, mientras que para las discontínuas desde el día en que el titular pudo ejercitarla y no lo hizo. No se computan los períodos de tiempo en los que la servidumbre no se podía ejercitar. D. $8,6,7$ :

7. Si sic constituta sit aqua, ut vel aestate ducatur tantum, vel uno mense, quaeritur, quemadmodum non utendo amittatur, quia non est continuum tempus, quo, quum uti non potest, non sit usus. Itaque et si alternis fannis vel mensibus quis aquam habeat, duplicato constituto tempore amittitur; idem et de itinere custoditur; si vero alternis diebus, aut die toto, aut tantum nocte, statuto legibus tempore amittitur, quia una servitus est. Nam et si alternis horis, vel una hora quotidie servitutem habeat, Servius scribit, perdere eum non utendo servitutem, quia id, quod habet, quotidianum sit.

Otra cuestión será cuando la naturaleza de la servidumbre es de las llamadas negativas, por una indiscutible analogía, el inicio del cómputo se realizará desde el día en el que se ha verificado un hecho que impida su ejercicio, esto es, desde que el dueño del predio serviente realiza algún acto contrario al contenido mismo de la servidumbre. 
Una muestra más de la evidente influencia del Derecho Romano en nuestro Código Civil viene reflejada en el art. 546 párrafo segundo (19) "Este término principiará a contarse desde el día en que hubiere dejado de usarse la servidumbre respecto a las discontínuas y desde el día en que haya tenido lugar un acto obstativo a la servidumbre respecto a las contínuas" (20).

Sólo una vez que concurren estos cuatro requisitos el non usus, superando la clásica estabilidad que genera el paso del tiempo, se convierte en un modo, aunque indirecto, plenamente eficaz para la extinción de los derechos de servidumbre.

\section{NOTAS}

(1).- Como génesis jurídica en preferible hablar de constitución que de adquisición. Aunque si observamos a la servidumbre como un bien común del tráfico económico, por esa intrínseca adhesión al fundo al que pertenece, puede utilizarse el término adquisición de la servidumbre al hablar de su transmisión.

(2).- El término "bestellung" tiene un significado paralelo a "establissent" y recoge con exactitud el matiz constitutivo.

(3).- La utilización del término y del concepto de prescripción, como forma útil de extinción, crea una ambigüedad en el leguaje aún vigente en nuestros días. Nuestro Código Civil con frecuencia utiliza expresiones distintas para significar un mismo instituto. Por extinción se concluye el usufructo en el art. 513. 7; mientras en el art. 546. $2^{\circ}$ se habla de "no uso". Ver Albaladejo, Instituciones de Derecho Civil, parte general, 1960, pág. 482; Castán Tobeñas, Derecho Civil Común y Foral, parte general I, vol 2, págs. 854-856; y fundamental la monografía "De la prescripción extintiva", de Alas, de Buen y Ramos, Madrid 1918, pág. 87 ss.

(4).- La utilización terminológica de "no uso" es la literal traducción del concepto que se encuentra en las distintas fuentes romanas; en ellas siempre encontramos, al no existir ni el término ni el concepto de prescripción, los términos "non uti" y "non usus" como hecho general extintivo.

(5).- Como nos indica Grosso "Sulla servitú di non concurrenza e sul non uso delle servitú" en "R. Dir. Común" 1949, 2 y Branca "Non uso e prescrizione" en Scritti in onore Ferrini, Milán 1941,1 , págs. 169 y ss.

(6).- Son numerosos los pasajes de las fuentes que nos recuerdan esta particular fuente de extinción:

D. $8,3,18$

D. $8,5,9$

D. $8,6,2-4-6-7$

D. $39,3,17 \mathrm{pr}$.

D. $43,20,1,23$

D. $43,20,1,5,1$

C.I. $3,34,10$

(7).- Através de la distinción que nos ofrece Gayo, recogida en D. 8, 2, 6, en las servidumbres urbanas el mero no uso de la servidumbre no es considerado, a fines de extinción, suficiente si no se encuentra en concurrencia de la usucapio libertatis.

En realidad el non usus y la usucapio libertatis no son institutos muy distintos, sino dos aspectos de un mismo hecho; en el no uso se considera la falta de ejercicio de la servidumbre por parte del titular, y en la usucapio libertatis se centraba la cuestión desde la perspectiva del gravado por la servidumbre. El non usus sería el presupuesto y la usucapio libertatis la consecuencia jurídica. 
(8).- Este límite de tiempo que se indica en la Ley decemviral es también propio de la época clásica como nos atestigua Paulo Sent. X, 17, 1-2.

(9).-Interesante por la correlación entre C.T. 4, 14, 1 y C.I. 7, 39, 3.

3. Sicut in rem speciales, ita de universitate ac personales actiones ultra triginta annorum spatium minime protendantur. Sed si qua res vel ius aliquod postuletur, vel persona qualicunque actione vel persecutione pulsetur, mihilominus erit agenti triginta annorum praescriptio metuenda; eodem etiam in eius valente persona, qui pignus vel hypothecan non a suo debitore, sed ab alio per longum tempus possidente nititur vindicare.

(10).- C. I. 3, 34, 13:

Sicut usumfructum, qui non utendo per biennium in soli rebus, per annale autem tempus in mobilibus vel se moventibus diminuebatur, non passi sumus huiusmodi sustinere compendiosum interitum, sed et ei decenni vel viginti annorum dedimus spatium, ita et in ceteris servitutes non utendo amittantur non biennio, quia tantummodo soli rebus annexae sunt, sed decennio contra praesentes vel viginti annorum spatio contra adsentes, ut sit in omnibus huiusmodi rebus causa similis, differentiis explosis.

(11).- Nuestro C.c. en su art. 537 no observa la distinción dentro de la usucapión entre presentes y ausentes, seguramente por influencia del 546 (influenciado por el 1073 C.c. italiano) -que abunda en lo ya indicado por el 537-, intentando evitar que a "sensu contrario" el no uso por 10 ó 20 años hiciera generar una servidumbre. Algo, sin embargo, perfectamente factible a través del instituto de la quasi possessio iuris, en este sentido Fermín Camacho de Los Rios, "Supestos de quasi possessio iuris", Granada Serie Maior, págs. 215 y ss.

(12).- Incluso dentro de la pandectística se mantiene la distinción entre non usus y prescripción, como comprobamos en Windscheid Pandette 1, 2 págs. 309 y ss., así como 426 ss. que nos informan de dos institutos diversos "Verjailhrung" y "Auspruch".

(13).- Grosso, "Sull le genesi storica della estinzione delle servitú per non uso" en Faro it. 1937,4 , págs. 266 y ss.

(14).- Sólo una excepción a esta imposibilidad de extinción por non usus parcial, la indicada por Paulo en D. 8, 4, 6:

Iter sepluchro debitum non utendo nunquam amittitus.

(15).- Biondi Biondo "La servitú prediali nel diritto romano", Milán 1969, pág. 322.

(16).- También se opina en contra, apoyandose en D. 43, 16, 10 donde Gayo indicaba que solamente expulsado por los propietarios, el usufructuario tiene derecho a proceder por el daño. Análogamente indica Biondi que se consuma la perdida aún cuando el non usus nazca de impedimento violento del propietario del fundo sirviente, a tenor de su particular interpretación de D. 41, $3,4,26$.

(17).- Amelotti, "La prescrizione delle azioni in dir. rom", Milán 1958, 211 y ss.

(18).- En atención al C.I. 7, 40, 2, debemos considerar que en el Derecho justinianeo se considera válida la interrupción civil, generada mediante acción o denuncia judicial relativa a la propia servidumbre.

(19).- Este inicio del período extintivo también se encuentra recogido con una patente influencia romanística en la Ley 406 de la Compilación Navarra, donde también se habla de acto obstativo, pero con referencia a las servidumbres negativas, así como la Compilación Catalana en su art. 344.

(20).- Auque nuestro Código recoge los supuestos de servidumbres contínuas y discontínuas nada dice de las servidumbres negativas. 